$\xi=-1$

\title{
Implementation methodology for android application development
}

\author{
Bulli Babu $^{1 *}$, Jada Naveen², Kondaveeti Rajinikanth ${ }^{3}$, Pothagani Sai Ram Krishna ${ }^{4}$ \\ ${ }^{1}$ Associate Professor, Department of Electronics and computer Engineering, KLEF Deemed to be University \\ ${ }^{2}$ Department of Electronics and computer Engineering, KLEF Deemed to be University \\ ${ }^{3}$ Department of Electronics and computer Engineering, KLEF Deemed to be University \\ ${ }^{4}$ Department of Electronics and computer Engineering, KLEF Deemed to be University \\ *Corresponding author E-mail:navi2uforever@gmail.com
}

\begin{abstract}
As the tuning flow of the modern world of technology, the mobile APKs are tremendous extracting segment of the globalization smart marketing technology. smart APKs applications are involving to a meteor pace to rise level of $\mathrm{n}$ utilizes and users a rich in report. AS on the paper, Android Platform for the developing the mobile applications. Firstly, we are Here To explain about the develop the android application as by our learnt knowledge we have created the application According to my college Website as the Business users pattern mode. Majorly we have to describe in this app is website users have to use the systems to check the recent updates and announcements and other regarding the things about related to the academic details. for that sake we have created the application by the tutorial knowledge and basics. This app can used as same as website with as the single tap in the smart mobile. As we are tried the app as best as possible manner in our way of our knowledge. This knowledge and creation of our works we have learnt lots things .I acquired good progress report in the creation of the application.
\end{abstract}

Keywords: Android platform; Application Frame work; Android Run time.

\section{Introduction}

Android is a new latest trending technology by the usage of smart mobiles. Completion time period of years, the word of mobile has been interchanged to the smart network technology. The condition has no large time gap of communication tool, even the condition became an dependent part from our daily life. Different categories and sectors of internationally connected with the teleacceptance approvals and most encrypted data to the smart technology. The consistence of the part is very great and it leads to android mobile developers.

My Android mobile application is prefer to the business sector. The prefer architecture source is addition in the operating technology, framework of application .The mobile applications is dependent on the source codes, as the allows developers to write codes in the source code language. The controls of mobile applications is based on the written of source code. The consider platform is bullet pointing notice to develop the APK on basis of goggle android SDK OS provided as the stack software of the manageable preferred techniques for development of application and all aspects is not maintained the use of the normal java. IDEs .As to explain about my mobile app is by using the platform of five simple steps the user can develop the application easily based on the steps. The client to provide the consumers with a vast choice of service. These services help the consumer to reach and contact the Business with ease and stay in touch with the business.

\section{Background study}

Android Architecture:

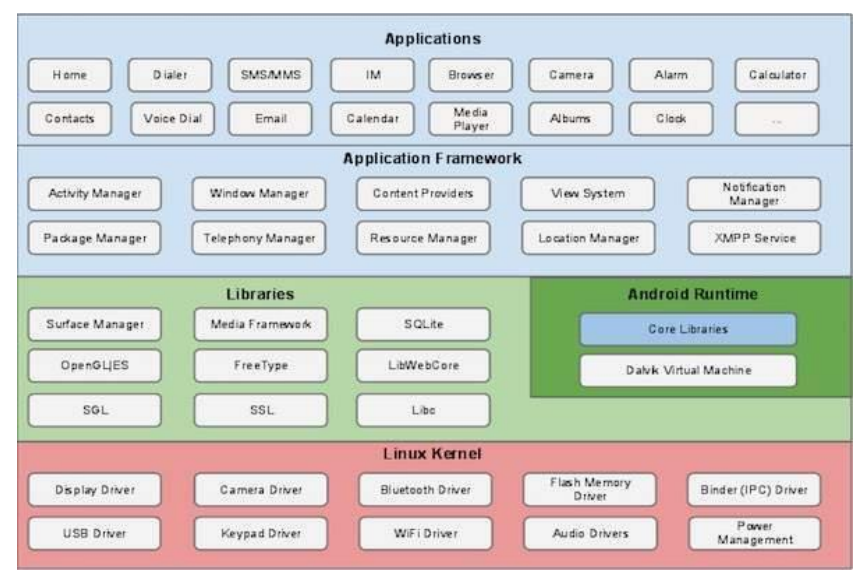

Android System is an intellectual outwards to the extracting for smart devices, this is primarily basis of which was announced to the world by goggle in the year of 2005 , android to be open with the world in 2007, with the open handset alliance of theoretically and practical by the machine works and communication companies dedicate to advance open standard for smart mobiles. New gen technologies are developing more and more applications to the world. 


\section{Related works}

The application gives the business user to provide the consumers with a vast choice of services. These services help the consumers to reach and contact the businesses with ease and stay in touch with business user. This establishes a communication channel between the user and, the business.

Basic/simple services: Basic or simple services are those types of services which provide just the basic information about a service. List of details:

- List of all the basic services

- Image that describes about a services.

Special services: Special services are those types of services that provide a detailed information about a service.

- List of all the services

- Simple description about the service

- A form for sending in a new service request

- History of the previous requests sent

Services with workflow: the service float will attains the accessibility of variation of the status to the service request send by the business consumer. Business user can also visualize the status position of the service request to the consumer and let them know the current position of the particular request.

- Add new service type with the desired group name for that service

- Add another new service that falls under a group

\section{Proposed System}

Proposed system main aim at developing an mobile application by the android based features of techniques that the expenditures had made the employment technology of different excavation sites are registered and it can be displayed at the any point of time by utilizing the android based mobile application the minute to minute the activities of the remote accessing database server construction site construction can be easily be updated construction database server. Initially the site organizer using his login credential has to login in the mobile application loaded in his device. After which has to upload to upload the recent work in progress report. using the web service data in the mobile device can be updated in the remote database. Thus the data in the accessed project as (management of system).The proposed work carried out the logical point can be easily be monitor use the system. the proposed work consists of various different types of advantages ,savage of time ,secure surrounding, will prevent to the work efficiency and corruption.

\section{Android Studio}

Android professionally integrated development environment(IDE) for development of the android platform. It was officially announce to the world in the year of 2013 at the venue of Google I/o convene. Android studio is a available in technology by the cost of free under the appliance of Apache License 2.0.Android is studio is free of cost available and primary access stage starting from the 0.1 in the same year, and then enter bet stage start from the stage 0.8 of first version outward to the world in the 2014,later that to recent nearly started to the same year and announced the 1.0 of the version depending on Jet Brain's, The SDK software is designed particular for development of android .this is Officially download any operating system an interchange of tools in the android development as primary IDE for goggle starting stage of development.

\section{Analysis}

Analytics play a major role in marketing a business. Analytics help the business users to easily analyse and estimate the buying patterns of a consumer. The Business user gets a wide variety of analytics with the application suite. They are majorly classified

Products based analytics: In the orientation of business it helps clients or users to easily analyse and estimate the buying the patterns of a consumer.

Order based analytics: As the marketing of business user can keep a track on the orders under the satisfaction level of consumers.

Analytics on the application: The Application based analysis is very important for business user.

Campaigns based analytics: The user can improve and improvise whilst creating a new campaign.

\section{Implementation Methodology}

Tools: Colour Pic, Icon Generator

Architecture Pattern: MVC(model view control)

Technologies: Java

Data Bases: Mysql, Sqlite

User interface: Html, Javascript

Transmission: SON/XML

MVC: Model View Control

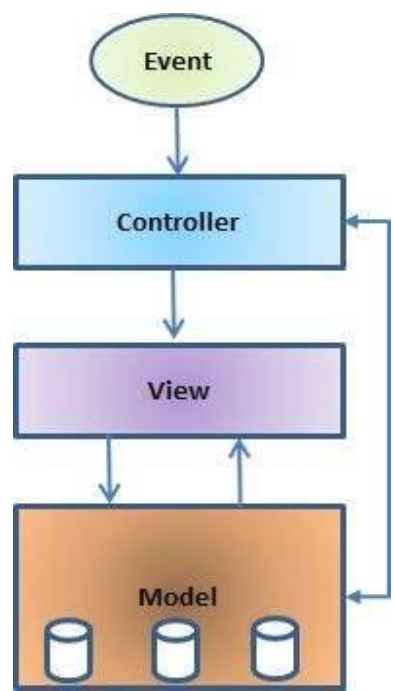

MVC is the interface layer and gives the rising factor to the separation of concerns. the controller will receives all requests for the works and the application and with design to the data needed by the displaying factor. The displaying factor can uses the data prepared by the controlled to evaporate a final presented response. 
Business use case Diagram:

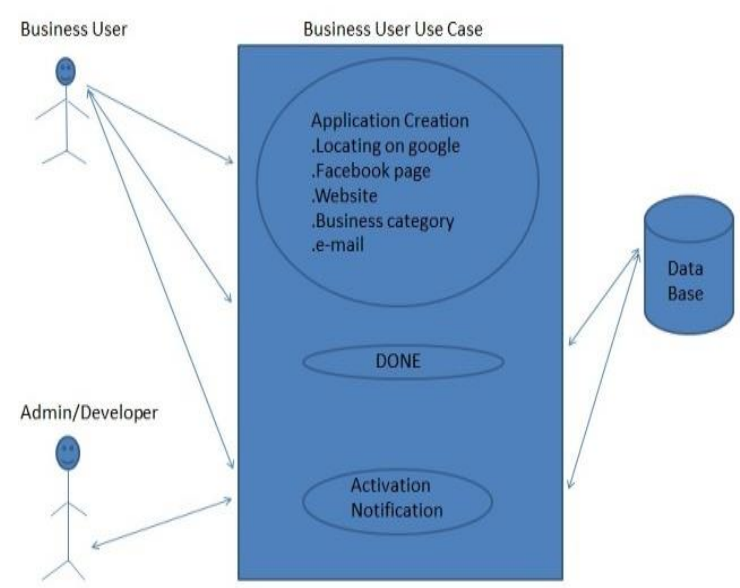

Level1: Business User creates his app using the FIVE STEPS and activation notification will be sent. Based on the permissions. it'll be activated by the admin or the user itself.

Level2: Here business user enters the data required after the app is activated. Admin plays a supporting role here, If there are any problems for Business User, he'll be dealing with them.

Level 3: This is actual usage of app by the consumers. Whenever Business user wants to interact with users, he broadcasts the notifications to the registered users. Admin will be playing supporting role here, If Business user needs any tech support or the app has a new update.etc

Class Diagram for Business User:

CLASS DIAGRAM FOR BUSINESS USER

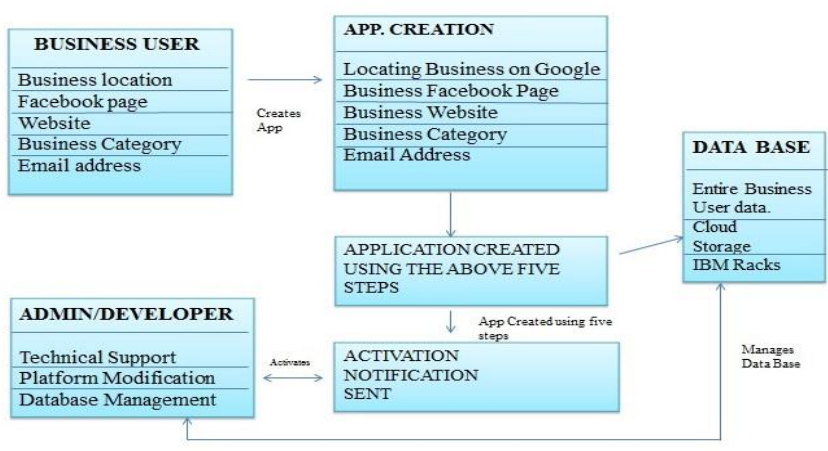

Business user contents the maintenance of Business Locations, Social Websites, Data Storage/Transferring Accounts are leads to Generate the Application. After the Creation of the application By Using All the services of the Five steps an Activation Notification will be sent Later it move on the Admin section for Technical support And then it resides to the Database management, Later it move on to the designation platform to the client to select categories in mean while about the type of business dealing to the link in the creation of the application. After the demo completion the client accept deal the work to do in demo progress.

\section{Technologies Employed}

Java: Java program that conciliates the reason of different based designation as on the one of the is concurrent the language in a utilize to build a different classes in it of library modules of techniques maintains in greater level usage. And the other one is class based and object oriented in specific manner to implemented in application "write once, run anywhere" the meaning that compiled source code and progress to execute the platform of the framework under no progress to execute need for the recompilation Java ap- plications are encrypt compiled to byte code that can execute on any Java virtual machine.

\section{JAVASCRIPT}

JavaScript is a raised level of the technology basis. It was untapped and external dynamic interpreting sourcing language. the current of the standard in the "ecma" language encrypted a long side of the hypertext markup language \&css .this is the three listed technologies of "www" of the production The maximum of web pages and sites employs on it. This is supporting from the new generation web browsers without plug-in. this format is a prototype dependent with the initial class response of the functions. By the creation of a various paradigm language, the suppose the factor of object orientation of the improvise and responding of the program execution. The API on the arrays of the time expressions in on order as the not to addition in any i/o database initial startups visualizations init based host surrounding field technique.

\section{HTML}

$\mathrm{Html}$ is similarly referred to create web pages on the browsing websites. The created WebPages can be displayed and audible to the users on the browsing sites.

The explains about the architecture of a website with the full cues for representation, when the creation of markup language, it is specific rather than a executing program of the language.

The Factorials we used the creation the blocks of the all websites. This language will accept the pictures and any objects to encrypt and In a mean while it helps to build the conversion relation form. This means to provide a design documents by noticing architectural semantics of the bullet points and any other items.

\section{JSON VIEWER}

JSON describes about the reading sentence in the source code to format of the page to describe to the normal visible to the homosapiens. The format of the object notation is to sending the data objects attribute pairs. This is the initially listed content from the non assign browser/server the content of extract to maximum of space inter places the xml.

Even the genuine the script from the java scripting language. This is the independent language of the format. Source the for creating json data is any time currently access to available in various programming languages. This json format was real specific by the douglascrockford. It is present position explains the standard, the ecma is minimum sentence completion of word end. This only allow to semantic and security reasons. the officially type for Json.

\section{Hibernate}

Hibernate is object linkup to the framework in the mapping process for the java language. The evaluation of providence of a frame work to map the object of the primary design to the traditional linkup manner in the database. The hibernate will resolves the object linkup impedance is syncing to the problems by the interchange to exactly to the persistence to linkup of the database to access the maximum level to over take the functions of the taken in the resulting manner.

\section{Conclusion}

The present paper will shows the android based platform development and application development by using the same platform by the databases. The based several techniques and develop the 
emulator and the developed application. The major dependent on the staring creation of the five simple steps of the lost host managing direction of the any secure confidential goggle login site and the creating the application by the several techniques as the java and php, relating techniques of the designation techniques of the application. After the creation application the checking server bugs in the apk databases in the web application .this web apps decide the category to the which relating sector to it is designed by the client. Finally completion the apk the demo is accepted by the client .It is acceptable permission by the appstore to place on it by the relating sector choose by us. As the resulting the successfully completion application.

\section{References}

[1] What is

android? http://developer.android.com/guide/basics/what-isandroid.html

[2] http://www.tutorialspoint.com/android/android_architecture.html

[3] http://www.slideshare.net/VijayRastogi/ppt2-introandroidarchitecturecomponentsd6

[4] Static detection of malicious code in executable programs by $\mathbf{J}$ Bergeron, M. Debbabi, J. Desharnais, M. M. Erhioui, Y. Lavoie, and N. Tawbi.

[5] Android Official Website (2008)—“Android | Official Website", <http://www.android.com/>.

[6] An Android Application Sandbox System for Suspicious Software Detection, by Thomas Bl“asing, Leonid Batyuk, AubreyDerrick Schmidt, SeyitAhmetCamtepe, and SahinAlbayrak

[7] www.blackhat.com Jeff Lessard Gary C. Kessler, "Android Forensics: Simplifying Cell Phone Examinations” Small Scale Digital Device Forensics Journal Vol. 4, No.1, September 2010 Issn\# 1941-6164,pp1-12.

[8] Tahat A.A. "Mobile personal electrocardiogram monitoring system and transmission using MMS"7thInternational Carbbean Conference on Devices, Circuits and Systems, 2008, 4:1-5.

[9] Feng Wang, Jiayu Zhu, MengShen, "A Communication Protocol of Wireless Sensor Network for Mobile Healthcare System" IEEE/ACM International Conference on Green Computing and Communications, pp 554-559, DOI 10.1109/GreenComCPSCom.2010.98.

[10] https://dspace.mah.se/bitstream/handle/2043/10721/AndroidApp licatcionDevelop-

ment.pdf?sequence $=1$ roidApplicatcionDevelopment.pdf?sequen $\mathrm{ce}=1$ 